\title{
Study on the Confronting Strategies of Infodemic from the Perspective of University Libraries: Case Study and Demonstration Based on 42 “Double First-Class” University Libraries
}

\author{
Guanxian Shan, Renyu Qian, Xiaotao Li \\ Library, Nanjing University of Aeronautics and Astronautics, Nanjing, China \\ Email: sgxlib@nuaa.edu.cn
}

How to cite this paper: Shan, G.X., Qian, R.Y. and Li, X.T. (2022) Study on the Confronting Strategies of Infodemic from the Perspective of University Libraries: Case Study and Demonstration Based on 42 "Double First-Class" University Libraries. Open Access Library Journal, 9: e8357. https://doi.org/10.4236/oalib.1108357

Received: January 10, 2022

Accepted: January 27, 2022

Published: January 30, 2022

Copyright $\odot 2022$ by author(s) and Open Access Library Inc.

This work is licensed under the Creative Commons Attribution International License (CC BY 4.0).

http://creativecommons.org/licenses/by/4.0/

\begin{abstract}
With the continuous spread of COVID-19 pandemic around the world, infodemic has been generated. People have realized the importance of confronting the infodemic together because of its threat. As the important places for information service, the university libraries play important roles in confronting the infodemic. This paper systematically analyzed the generation and propagation of infodemic, built the mechanism framework of confronting the infodemic by university libraries, and verified the framework by the case of 42 "Double First-Class" university libraries. The results of the web survey demonstrated that bibliotherapy, information therapy and information literacy education were effective measures to counteract infodemic.
\end{abstract}

\section{Subject Areas}

Library, Intelligence and Philology

\section{Keywords}

Infodemic, University Libraries, Bibliotherapy, Information Therapy, Information Literacy

\section{Introduction}

Since the end of 2019, the outbreak of COVID-19 has been occurring globally. At the same time, infodemic, which is anotherepidemic, started to spread around the world. Compared to the virus itself, the infodemic spreads at a faster speed with higher criticality. It not only brings unnecessary fear for the public, which 
increases the difficulty of medical rescue, but also potentially causes social chaos. Therefore, Tedros Adhanom Ghebreyesus, the Director-General of the World Health Organization (WHO) has suggested that the world should boycott the infodemic together and strengthen the effort to resist the infodemic [1]. China has been taking various measures in the prevention and control of COVID-19, disclosuring the epidemic information timely, efficiently, and transparently, as well as making great efforts in resisting infodemic jointly, which was recognized and praised by United Nations General Assembly Organization [2].

The university libraries in China played important roles in delivering the reliable resources on the infodemic, as well as providing online teaching resources. In the meantime, by the information literacy education, including information retrieval and information screening, the libraries are the vital force to dispel untrue epidemic information and normalize the information spreading [3] [4]. Nevertheless, the service emphasis shifts of university libraries still lagged in time when facing the sudden epidemic, which is hard to meet the needs for resisting the epidemic rapidly [5]. This also indicates that the university libraries have not yet formed the emergency service mechanism for the information and resources of significant epidemic at present. On that account, this paper explored the research topic on "How the infodemic is generated and spread and how can the university libraries tackle this issue?" This paper tried to reveal the process of infodemic generation and spread by the theory of the generation and spread of rumor and built the service mechanism framework for university libraries to tackle the infodemic.

\section{Infodemic Theories}

\subsection{Retrospect of the Connotation and Theory of Infodemic}

Infodemic was first interpreted by Dr. Briand, who is the department head of WHO. She pointed out that a bulk of plausible information was generated in social media accompanied with the outbreak of infectious disease. The rapid spread of these kinds of information made it hard for people to get reliable information and get proper guidance [6]. The concept of infodemic is novel, and even can be said that it is generated because of COVID-19 pandemic, leading to the interpretation and emphasis by the experts. Although the concept of infodemic was brought out by Karen et al. first [7], the infodemic phenomenon has been long existed. Briand thought that it accompanied the outbreak of each infectious disease, such as vast rumor during the period of yellow fever [8]. The essence of infodemic was so to speak the rumor related to epidemic.

The theoretical study on infodemic can be retrospected to the research on rumor. The theoretical study on rumor can be divided into the following areas: the definition of the rumor concept, the generation of rumor, and the motivation and the path of rumor spread. For the definition of rumor, Robert et al. defined rumor as the issue under discussion and information without official verification. Some of these kinds of information are true, while others are fake. It is 
generated with the issues related to current affairs and widely spread [9]. As for the reason for rumor outbreak, Allport et al. considered the generation of rumor was driven by the anxious emotion among the public, and is related to the criticality of the emergency and the degree of ambiguity of the information [10]. In the spread pathway of the rumor, Zhou et al. and other scholars thought it was not transmitted in the formal channels, while widely spread in the informal channels [11].

Combined with the aforementioned theoretical basis, "Vast information generated in social media, which is not verified officially, accompany with the occurrence of epidemic" was defined as infodemic in this paper. The wide spread of these kinds of information makes it hard for people to get reliable guidance.

\subsection{Theoretical Framework of the Generation and Spread of Infodemic}

Based on the definition of the connotation of infodemic, this paper divides its theoretical framework into three stages, including reasons for rumor generation, the spread pathways, and the results of outbreak. In the first stage, the framework for the reason of infodemic outbreak is built based on the rumor theory raised by Allport to analyze the essential elements of the infodemic generation and their influential relationship between each other. Based on that, this paper illustrates through which pathway the infodemic is spread. Finally, the results of infodemic outbreak are elucidated (see Figure 1).

\subsubsection{The Framework of the Reasons of Infodemic Outbreak}

Based on the rumor theory that was raised by Allport, the generation and spread of rumor depend on the following factors:

1) Psychological factors: the generation of rumor is driven by the inner anxiety and fear of individuals.

2) Social environmental factors: the extent of rumor spread depends on the criticality of the issues produced by drastic change in environment.

3) Information environmental factors: the degree of ambiguity of the authoritative information or the degree of the missing of the official information [12].

When the vital social crisis event occurs, people feel uncomfortable because of the drastic change in social environment. Since the lag or missing of official information, the insecurity of the information intensifies the inner anxiety among the public. In this case, rumors, which are essentially the protest for the turbulent external environment and missing official information, are generated.

\subsubsection{The Framework for the Pathway of Infodemic Outbreak}

The spread pathway of infodemic essentially needs the framework for information communication. Combined with the characteristics of the spread pathway of rumor, the framework for the pathway of infodemic outbreak is built based on the information communication framework established by the famous information scholar, Mihaylov, from the Soviet Union [13]. Different from the traditional 


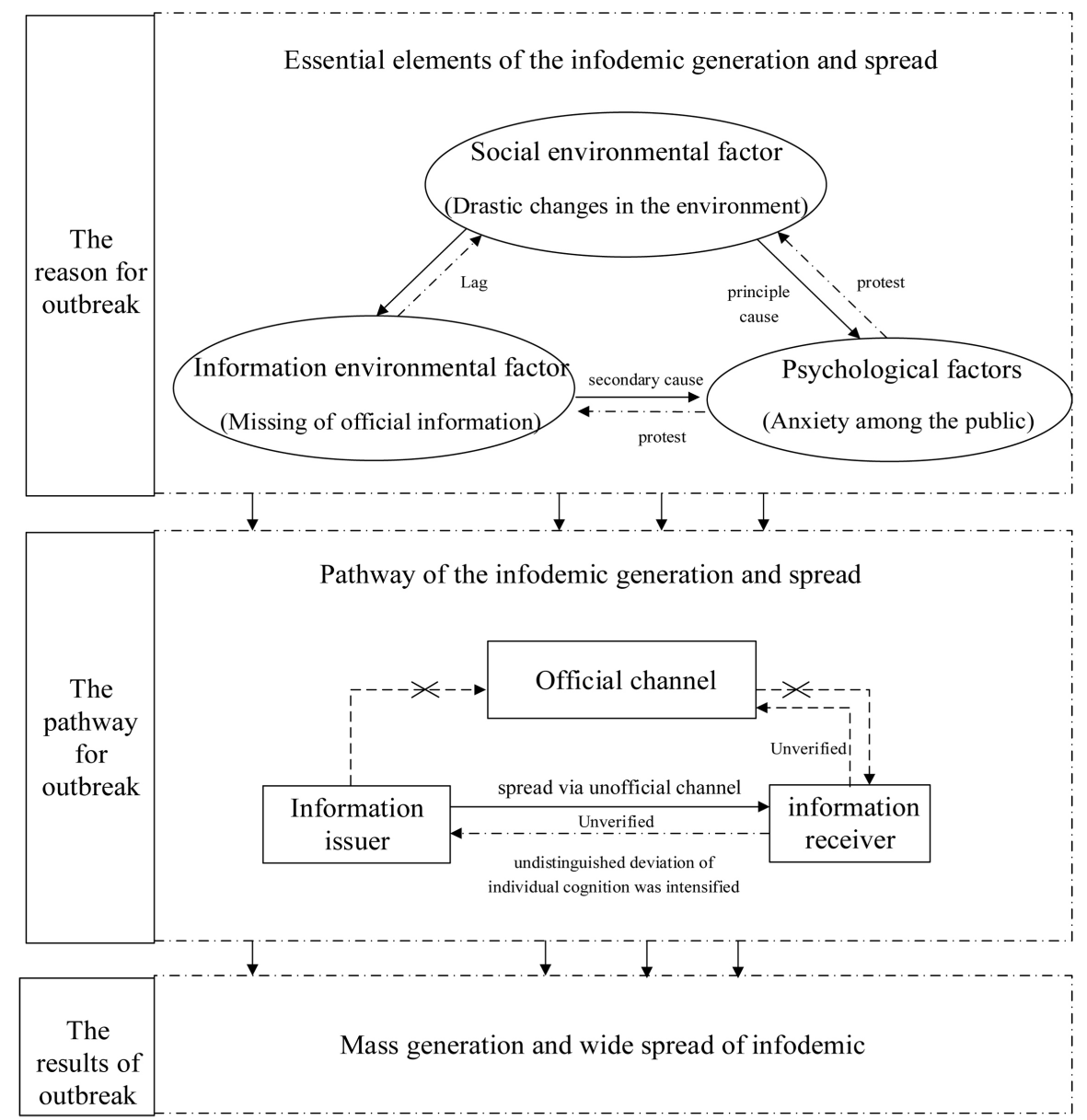

Figure 1. Theoretical framework of the generation and spread of infodemic.

information communication framework, the spread of rumor relies on unofficial channels, without participating in the spread in official channels.

When the infodemic is spread via unofficial channels, including Wechat Moments, Weibo, Twitter, the information receivers trust the information indistinguishably and they would not verify the information from the official channels since they are in the condition of inner anxiety, resulting in the cognitive bias of individuals, as well as accelerating the wide spread of infodemic.

\subsubsection{The Framework for the Result of Infodemic Outbreak}

The result of infodemic outbreak is the mass generation and wide spread of rumor related to the epidemic, leading to the difficulty for people to get reliable information guidance, even causing the unnecessary panic among the public and the society.

\section{Construct the Mechanism Framework for University Libraries to Confront Infodemic}

\subsection{Overall Analysis}

From the theoretical analysis of the generation and spread of infodemic, we can 
know that the drastic environmental change, the public psychological anxiety, and the ambiguous official information are the essentials for the generation of infodemic. In addition, the individual cognitive bias can aggravate the wide spread of infodemic in the spread pathway.

University libraries have the function of providing bibliotherapy psychological adjustment service, delivering reliable information and resources of epidemic, and improving the capability of information literacy. This is helpful for solving the issues resulted from the public psychological anxiety, the ambiguous official information, and the individual cognitive bias so that the infodemic outbreak can be restrained. While the issues raised by drastic environmental change are tend to be confronted from the macroscopic level, exceeding the function of university libraries in the middle level. In this regard, this paper explores from three levels, including psychological adjustment, information disclosure, and information literacy, to put forward the framework for service mechanism of university libraries to tackle infodemic (see Figure 2). In the meantime, by investigating and researching the websites and Wechat subscriptions for the 42 "Double First-Class" university libraries, the rationality and enforceability of the

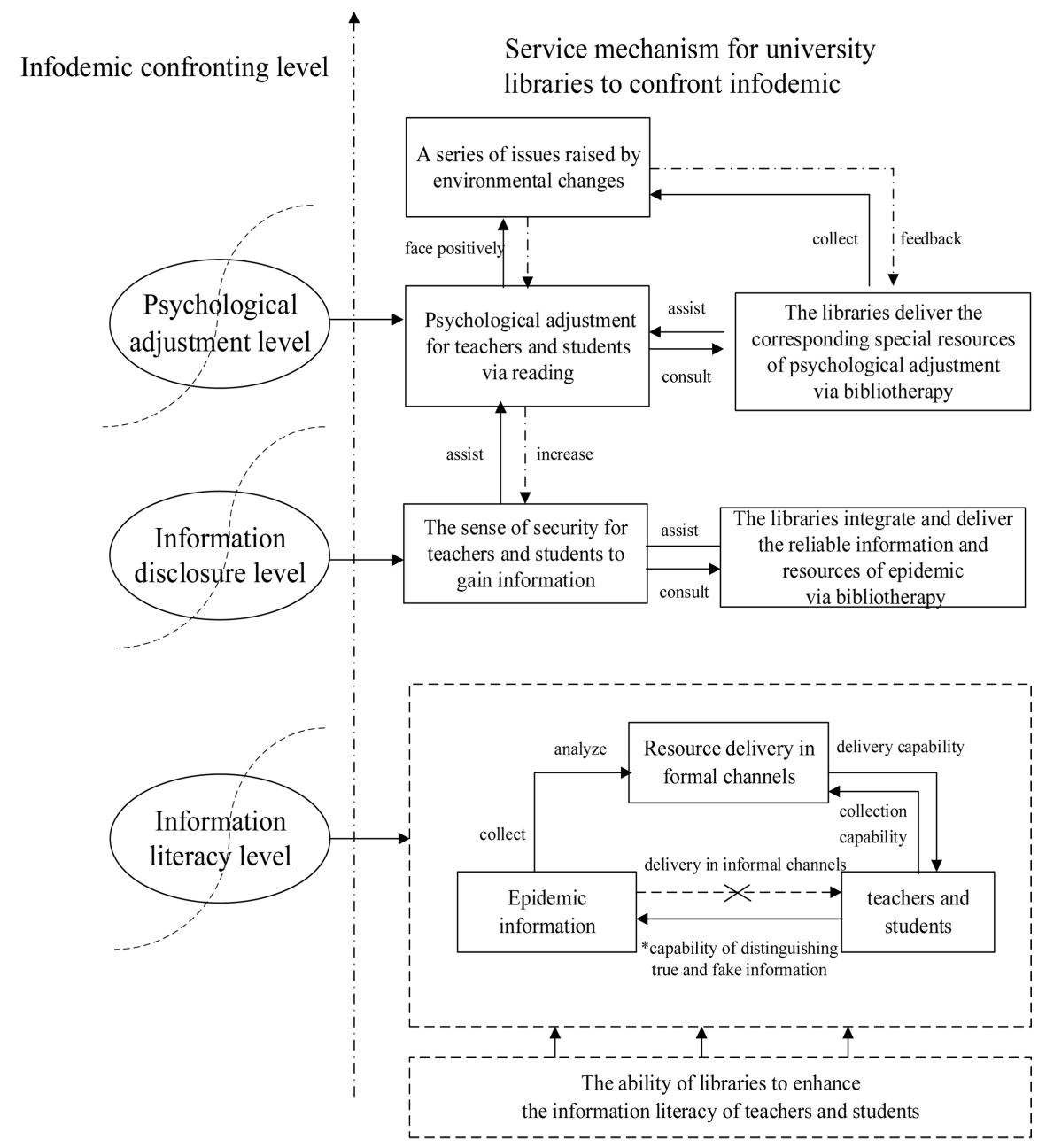

Figure 2. Mechanism framework for university libraries to confront infodemic. 
framework construction are verified. The investigation results are shown in Figure 3.

\subsection{Analysis of Service Mechanism in Psychological Adjustment Level}

\subsubsection{Analysis of Infodemic Confronting in Psychological Adjustment Level}

The generation of anxiety is not only originated from the epidemic itself, but also from the secondary issues caused by the epidemic, including course learning, thesis writing, graduation, job hunting, and family. When teachers and students are in the mood of anxiety, it is hard for them to make rational judgments for the matter, and they may even make "anxiety releasing" criticism on that matter. These anxious criticisms can easily resonate with other anxious people, which is the key for the generation and spread of infodemic.

Reading is the best company for adjusting the psychology. By utilizing the idea of bibliotherapy, university libraries make directional delivery of related books on special topic of psychology to realize the objectives of preventing and relieving unnecessary panic among audience [14]. By using this inner adjustment method of bibliotherapy, the possibility of anxious criticism made by teachers and students online can be reduced effectively so that the outbreak of infodemic can be prevented.

\subsubsection{Investigation Results in Psychological Adjustment Level}

Among the 42 universities, almost all of them use the strategy of bibliotherapy to relieve the inner pressure of teachers and students. However, there are only 15 university libraries (35.7\%) that deliver special resources aimed at possible psychological issues during the pandemic. For example, Sichuan University library customizes the tree-hole mailbox, which enables teachers and students to pour out the secrets in their hearts. Additionally, this library sorts out different book

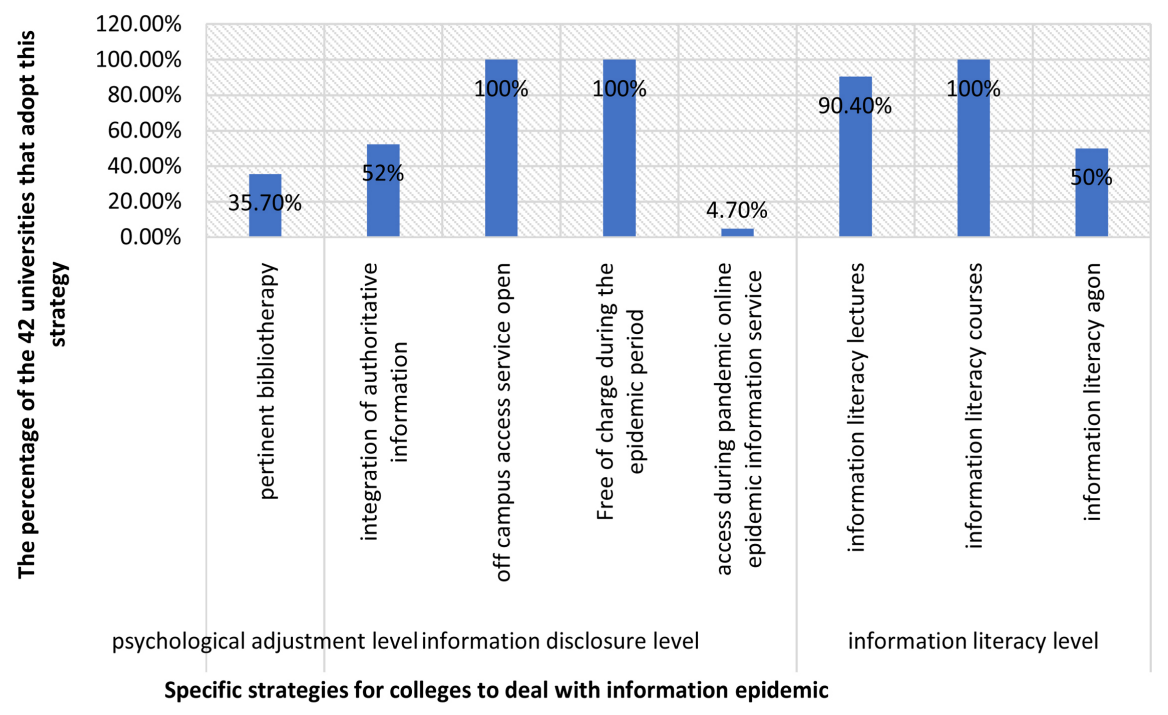

Figure 3. Investigation results on infodemic confronting of university libraries. 
lists according to the idea of bibliotherapy from four modules, including understanding emotions, defusing and adjusting, prevention and control in macro level, and stabilizing moods, letting the teachers and students to jump out from the anxiety via reading. As can be seen from the participation effects, bibliotherapy relives the inner anxiety to some extent, provides the space for teachers and students to express their inner words, resulting in the avoidance of the possibility of "releasing the anxious criticism" from other pathways.

\subsection{Analysis of Service Mechanism in Information Disclosure Level}

\subsubsection{Analysis of Infodemic Confronting in Information Disclosure Level} The opaque and undeclared authoritative information brings the public the sense of insecurity on information. Under the force of insecurity sense, people desire to fill in the blanks and fuzzy shadows of information, which providing opportunities for the outbreak of various rumor. In the period of severe epidemic, teachers and students have strong desire for knowing the truth of epidemic to fulfill their sense of security of information. This kind of sense of information security is not only originated the information public from government, but also relies on different kinds of information therapy from library to assist the disclosure of the information. By conducting investigation, it is found that the information therapy methods of university libraries include integrating the authoritative resources on epidemic, providing access service on epidemic resources off campus, offering open access to the epidemic resources during the pandemic, as well as providing consulting services on information related to the epidemic.

Indeed, information therapy is the important means to decrease the sense of insecurity on information of teachers and students by university libraries, which plays a role of "magnifier" in the communication of authoritative information. The libraries assist teachers and students to get reliable information about epidemic by performing various information therapy. This helps to enhance the sense of security of teachers and students, build the trustable media for information spread, and vanish the rumor.

\subsubsection{Investigation Results on Infodemic Confronting in Information Disclosure Level}

42 university libraries have adopted different information therapy to build trustworthy information spread media and information environment, reducing the infodemic risk caused by ambiguous official information. There are 22 (52\%) university libraries integrating the authoritative infodemic information. For example, Tsinghua University integrates the resources of epidemic from five angles, including authoritative academic journals, database, countries, research institutes, and patents. Almost all universities provide access service off campus by VPN and CARSI technology. Under the support from the database providers, almost all universities provide open access for electronic resources for a limited time during the pandemic. Several universities also provide online consulting 
service for information related to epidemic via Wechat and QQ. Among them, the most representative example is Zhengzhou University library. This library relies on "Resource bank of Q\&A on COVID-19 pandemic" to connect university intelligent service platform via eight channels. Teachers and students can get the latest information of epidemic by typing in key words in the subscription of the library, which realizes the accurate response to the questions on the epidemic so that effectively reduces the spread of infodemic in unofficial channels.

\subsection{Analysis of Service Mechanism in Information Literacy Level}

\subsubsection{Analysis of Infodemic Confronting in Information Literacy Level}

Under the condition of cognitive bias among individuals, teachers and students are unable to distinguish fake information, and even take the false information as correct guide, which brings unnecessary trouble for themselves. The basic solution for the individual cognitive bias is to improve the information literacy of teachers and students. By putting up information literacy lectures on epidemic, setting up live courses for information literacy on epidemic, and holding online agon, university libraries enhance the awareness of teachers and students on information utilization, intensify the collection, distinguishing, and delivery of information related to epidemic. Multipath information literacy education has direct and effective impact on preventing the outbreak of infodemic.

\subsubsection{Investigation Results on Infodemic Confronting in Information Literacy Level}

42 university libraries have adopted various means as the basic way to prevent infodemic outbreak. 38 (90.4\%) university libraries carry out information literacy courses on epidemic via the means of live streaming, with the emphasis of the courses on instructing information utilization techniques. 42 (100\%) university libraries utilize the methods of live streaming, short video, courseware download to conduct lectures on information literacy. The contents of the lectures involve preventing the risk of infodemic outbreak, including how to search for effective information on epidemic, how to discriminate the fake information, how to utilize information, and solving difficult problems in life. 21 (50\%) university libraries adopt the means of agon to promote the increase of the information literacy capabilities of teachers and students. For instance, Fudan University carries out the competition on "battle of data", which performs in-depth analysis of COVID-19 data, making the infodemic confronting more efficient.

\section{The Study on Infodemic Confronting of University Libraries}

\subsection{Pertinent Bibliotherapy Is the Main Approach of Relieving Infodemic}

Pertinent bibliotherapy is to do precise promotion on books according to the psychological features of teachers and students during the pandemic, in order to adjusting psychological mood of more audience and reduce the possibility of re- 
leasing their "anxious criticism" through other pathways. University libraries have realized that bibliotherapy is an important means to relive the inner pressure of teachers and students. However, the precise promotion service is lack for their psychological features during the pandemic in real-time applications. For this, university libraries reinforce the precise promotion service for the special book on the current basis. For instance, by performing the in-depth analysis of many anxious psychological features like the panic to the epidemic itself, and the implicative pressure for employment or the preparation for the postgraduate exam, the reading service can be promoted precisely for teachers and students with possible psychological problems.

\subsection{Open Information Therapy Is an Important Method to Prevent Infodemic}

Information therapy refers to provide reliable, free, and open information and resources on epidemic through varies methods so as to assist the disclosure of information and reduce the infodemic caused by the sense of insecurity of information. In critical incident, the sense of information security of public would be lowered if the authority tries to cover up the facts, which may aggravate the risk of infodemic. Adversely, by uncovering the truth openly and transparently can let the public realize a reliable information platform. Libraries, as the resource center for vast reliable information, is the best media for assisting the disclosure of authoritative information. During the pandemic, libraries should adopt more open information therapy, like increasing the time for open access to the electronic resources, providing more convenient services for off-campus access, to enable that the authoritative information and resources on epidemic can be delivered to teachers and students more conveniently and effectively.

\subsection{Improving Information Literacy from Different Angles Is the Basic Pathway for Infodemic Confronting}

For now, the emphasis of libraries is on the cultivation in the level of information utilization techniques, including how to search for effective information on epidemic, how to distinguish fake information, and how to utilize high-quality resources and information, while they may ignore if the forwarded information meets the requirements of ethics, morality, and law. Some teachers and students may be familiar with the techniques for information utilization, but they may not realize the responsibility of the conveyed contents in information communication. Therefore, university libraries should strengthen the education on information ethics and information morality via various means of lectures, courses, or competitions to enhance the social responsibility of teachers and students in information.

\section{Conclusion}

By sorting the related literature, this paper forms the theoretical framework for the generation and spread of infodemic and illustrates the reasons for its genera- 
tion and spread. On this basis, the mechanism framework is constructed for university libraries to confront infodemic in psychological adjustment, information disclosure, and information literacy levels. By investigating the cases of infodemic confronting of 42 Double First-Class university libraries, we verify the improvement of bibliotherapy, information therapy, information literacy in the three levels is the effective measure for confronting infodemic. At last, the specific strategies are put forward for how the university libraries should confront infodemic in the future.

\section{Fund}

This work was supported by Jiangsu province higher education digital library program (Grant No. 2020KT11).

\section{Conflicts of Interest}

The authors declare no conflicts of interest.

\section{References}

[1] Lancet, T. (2020) COVID-19: Fighting Panic with Information. Lancet, 537. https://doi.org/10.1016/S0140-6736(20)30379-2

[2] Xinhua (2020) UN General Assembly President Urges Avoiding Rumors about Coronavirus, Emphasizes Solidarity. http://www.xinhuanet.com/english/2020-02/09/c 138767676.htm

[3] Chen, Y.Z. and Xiao, W. (2020) Novel Coronavirus Pneumonia Emergency Information Service: Library in Action. Library Work in Colleges and Universities, 2, 2.

[4] Ahmed, T., Yang, C., Yang, H., Rahoo, L., Mahmood, S. and Poudiougo, B. (2021) Leadership Styles and Employees' Productivity: A Case Study of Public University Libraries of Jamshoro City, Sindh Province, Pakistan. Open Journal of Leadership, 10, 230-240. https://doi.org/10.4236/ojl.2021.103015

[5] Cai, Y.C. and Wu, Z.R. (2020) Information Resources Emergency Guarantee System of University Library. Library Journal, 5, 43-54.

[6] World Health Organization (2020) Novel Coronavirus (2019-nCoV) Situation Report-13.

https://www.who.int/docs/default-source/coronaviruse/situation-reports/20200202sitrep-13-ncov-v3.pdf?sfvrsn=195f4010 6

[7] Karen, H. and Tanya, B. (2020) The Coronavirus Is the First True Social-Media "Infodemic".

https://www.technologyreview.com/s/615184/the-coronavirus-isthe-first-true-social -media-infodemicl

[8] Erku, D.A., Belachew, S.A., Abrha, S., et al. (2021) When Fear and Misinformation Go Viral: Pharmacists' Role in Deterring Medication Misinformation during the "Infodemic" Surrounding COVID-19. Research in Social and Administrative Pharmacy, 17, 1954-1963. https://doi.org/10.1016/j.sapharm.2020.04.032

[9] Ju, Y.N. (2013) Principles of Public Relations. 5th Edition. Fudan University Press, Shanghai.

[10] Allport, G.W. and Postman, L.J. (1947) The Psychology of Rumor. Holt and Company, New York. 
[11] He, X.M. (2015) Modern Public Relations. Fudan University Press, Shanghai.

[12] Jiang, S.J. (2020) Information Novel Coronavirus Pneumonia Epidemic Rumors Spreading and Coping Research. Studies on Science Popularization, 1, 70-78.

[13] Mikhailov (1980) Science Communication and Information Science. Science and Technology Press, Beijing.

[14] Hu, J., Jiang, X. and Jiang, T.T. (2017) Research on the Practice of Reading Therapy for College Students Based on the Improvement of EQ-Taking the Construction of "Muxin Hut" in Sichuan University Library as an Example. Library Development, 3, 48-54. 